\title{
Selective forces acting during multi-domain protein evolution: the case of multi-domain globins
}

\author{
Joana Projecto-Garcia ${ }^{1,2^{*}}$, Didier Jollivet ${ }^{1,2}$, Jean Mary ${ }^{1,2}$, François H Lallier ${ }^{1,2}$, Stephen W Schaeffer ${ }^{3}$ \\ and Stéphane Hourdez ${ }^{1,2}$
}

\begin{abstract}
Multi-domain proteins form the majority of proteins in eukaryotes. During their formation by tandem duplication or gene fusion, new interactions between domains may arise as a result of the structurally-forced proximity of domains. The proper function of the formed proteins likely required the molecular adjustment of these stress zones by specific amino acid replacements, which should be detectable by the molecular signature of selection that governed their changes. We used multi-domain globins from three different invertebrate lineages to investigate the selective forces that acted throughout the evolution of these molecules. In the youngest of these molecules [Branchipolynoe scaleworm; original duplication ca. 60 million years (Ma)], we were able to detect some amino acids under positive selection corresponding to the initial duplication event. In older lineages (didomain globin from bivalve mollusks and nematodes), there was no evidence of amino acid positions under positive selection, possibly the result of accumulated non-adaptative mutations since the original duplication event (165 and $245 \mathrm{Ma}$, respectively). Some amino acids under positive selection were sometimes detected in later branches, either after speciation events, or after the initial duplication event. In Branchipolynoe, the position of the amino acids under positive selection on a 3D model suggests some of them are located at the interface between two domains; while others are locate in the heme pocket.
\end{abstract}

Keywords: Positive selection, Hemoglobin, Nematodes, Molluscs, Annelids

\section{Background}

The increasing number of sequenced genomes has revealed that the fraction of proteins with two or more domains represents up to $70 \%$ of the genes in eukaryotes, and tandem repeats in particular represent up to $20 \%$ of all sequences in multicellular organisms (for reviews, see Björklund et al. 2005; Han et al. 2007). Both duplication and adaptive evolution have been implicated in the origin and diversity of multi-domain proteins (Vogel et al. 2005). From a limited set of initial domains, the duplication and shuffling of domains has permitted the emergence of numerous and complex proteins with potentially novel functions. Although evolutionary events affecting

\footnotetext{
*Correspondence: jucpgarcia@gmail.com

1 CNRS UMR 7144, Station Biologique de Roscoff, Places Georges Teissier, 29680 Roscoff, France

Full list of author information is available at the end of the article
}

the domains' active sites are crucial to determine a proper functioning of the protein, mutations located on the exposed regions of the different domains and at their interface might play an equally important role by affecting folding and interactions between domains (Han et al. 2007). Very few studies have addressed how domains interact to produce the most efficient folding of multidomain proteins as each domain is usually considered independently, except when the active site is located at their interface (Han et al. 2007; Bhaskara and Srinivasan 2011). Proteins comprised of tandem-repeats of wellknown domains could be used to test the mechanisms that are involved in multi-domain proteins evolution to maintain a proper function.

The globin family is one of the most extensively studied protein families, especially in vertebrates. Functional tandem multi-domain globins have so far surprisingly only been found in invertebrates and prokaryotes (Weber and

\section{Springer}


Vinogradov 2001). Invertebrate multi-domain globins have been the target of numerous studies mostly focused on their primary and quaternary structures (Weber and Vinogradov 2001). In addition to the determination of their structure, studies aimed at understanding the evolutionary mechanisms of the origin of the domain repetition. They have shown that unequal crossing-over is the most common mechanism for globin gene duplication (Dewilde et al. 1999; Kato et al. 2001; Naito et al. 1991). Among invertebrates, only nematodes, mollusks, crustaceans, and annelids possess globin subunits with more than one functional protein domain (Weber and Vinogradov 2001) with a great structural diversity.

Nematode hemoglobins (Hbs, i.e. circulating globins) can be single-domain or di-domain (see Weber and Vinogradov 2001 for a review). These latter ones occur in the pseudocoelomic cavity, and assemble into octamers in the pig intestinal parasite Ascaris suum (Darawshe et al. 1987; De Baere et al. 1992). The globin gene diversity in nematodes is very large, and intron insertions and gene duplications were frequent in the nematode globin evolutionary history (Blaxter 1993; Hoogewijs et al. 2008; Hunt et al. 2009). However, di-domain Hbs are only known in the gut parasite ascarids A. suum and Pseudoterranova decipiens (Darawshe et al. 1987; Dixon et al. 1991).

Mollusks can exhibit both hemocyanins and Hbs, the latter being associated with species from hypoxic and fresh-water environments. Mollusk multi-domain $\mathrm{Hbs}$ can be found in bivalves and gastropods (see Weber and Vinogradov 2001 for a review). Bivalves of the genus Barbatia possess an intracellular di-domain $\mathrm{Hb}$ that forms high-molecular weight complexes (Grinich and Terwilliger 1980; Suzuki and Arita 1995; Suzuki et al. 1992). Other bivalves from the families Astartidae and Carditidae have more complex extracellular multi-domain $\mathrm{Hb}$ with at least 14-28 domains and gastropods from the family Planorbidae can also have multi-domain $\mathrm{Hb}$ with at least 10 domains (Weber and Vinogradov 2001).

In crustaceans, multi-domain $\mathrm{Hbs}$ are known in the water fleas Daphnia (Dewilde et al. 1999), and Moina (Kato et al. 2001), as well as in the brine shrimp Artemia (Von Brand et al. 1950), and all are extracellular. Artemia represents another example of multiple duplications almost as spectacular as the molluscan multi-domain $\mathrm{Hbs}$. This extracellular $\mathrm{Hb}$ possesses nine domains (Manning et al. 1990) and assembles into dimers (Jellie et al. 1996; Matthews et al. 1998).

Typical annelid extracellular globins are renowned and widely studied because of their amazing quaternary structure, which is comprised of a 3.6 MDa hexagonal bilayer (HBL) complex of globins and linker chains (see Weber and Vinogradov 2001 for a review). Annelid extracellular multi-domain globins have so far only been found in Branchipolynoe, a hydrothermal-vent endemic genus in the family Polynoidae (Hourdez et al. 1999a). In this genus, the $\mathrm{Hb}$ is extracellular, and corresponds to dimers and trimers of tetra-domain globins (Hourdez et al. 1999a). Based on sequence data, Projecto-Garcia et al. (2010) showed that these globins were closely related to single-domain intracellular globins, and were the result of successive tandem gene duplications.

The rare and sporadic occurrence of multi-domain globins in various invertebrate groups indicates multiple origins for these proteins, and suggests a possible selective mechanism for their formation under different environmental circumstances. These are usually linked to the need of organisms to possess complex pigments (not excretable) able to bind a maximum of oxygen molecules when subjected to hypoxia. Functioning as a multi-domain protein potentially requires structural modifications, and the knowledge of the globin tertiary and quaternary structures makes multi-domain globins good candidates to investigate the role of key amino acid replacements in the folding and interdomain interfaces of the protein. In this paper, we studied the selective processes that shaped multi-domain globins, from the initial duplication event to the present-day molecules and which structural modifications these globins underwent until today. We used the annelid Branchipolynoe, the blood clam Barbatia, and ascarid nematodes, three distinct taxonomic groups in which comparisons between a single-domain and multi-domain genes where possible (occurrence in sister species), to determine whether there was a common mechanism that shaped their structural evolution. These three hemoglobins evolved between 245 and less than 60 million years (Ma) ago, for the ascarids and the polynoids, respectively. Using maximum likelihood approaches, we determined which selective forces were acting during the evolution of the multidomain state and, in particular, identified amino acid positions under positive selection to understand their potential function. These amino acids were placed on a 3D model of each globin to propose structural interpretation of these amino acids.

\section{Results}

Only multi-domain globin sequences for some mollusks, annelids, and nematodes were retrieved from Genbank. Other multidomain globins from invertebrates (arthropods and Biomphalaria glabrata) did not meet the selection criteria for the $\mathrm{Hb}$ sequences (see "Methods" for details).

We were particularly interested in detecting a putative occurrence of codons under positive selection on the branches leading to the formation of the first duplication of the multi-domain globins, but also in all subsequent 
branches with $\omega$ greater than 1 (for which positive selection is suspected), where $\omega$ is the ratio of nonsynonymous to synonymous mutations $\left(d_{\mathrm{N}} / d_{\mathrm{S}}\right)$ (Fig. 1$)$.

\section{Selective pressures acting in multi-domain globin evolution}

Tetra-domain extracellular globin in Branchipolynoe: Positive selection before duplication

Using the single-domain sequences as outgroup, the maximum likelihood tree (Fig. 1a, see "Methods" for details) for the polynoids corresponded to one of the two topologies (topology B) established by Projecto-Garcia et al. (2010). This topology was used as a reference for the following maximum likelihood analyses on coding sequences using the codon model of substitutions.

The likelihood ratio test $(\mathrm{LRT}=19.05, \mathrm{df}=16$, $\mathrm{p}=0.25$; Table 1) with the branch model indicates that the 'free-ratio' model did not better fit the data than the 'one-ratio' model, indicating that the selective pressure did not differ markedly among branches. Most of the evolution of these sequences was characterized by purifying selection $(\omega=0.278)$. This branch model, however, is based on the comparison of the average $\omega$ ratio over the whole sequence and if only a few amino acid positions significantly differ, this test may not be able to detect it (Yang 2008). Similarly, the site model, that compares all positions averaged over all the branches, did not reveal any position that had a $\omega$ significantly different from the others (Table 1). Overall, most of the positions are under moderate purifying selection $(\omega=0.177)$, although $21 \%$ of the positions appear to behave nearly neutrally $(\omega=1$; Table 1$)$. As selective pressures can also greatly vary between sites on a specific branch, we proceeded with the branch-site model to test individual amino acid position along each branch. For the branch that leads to the first duplication (branch $a$, Fig. 1a), the likelihood values of the M1a and MA showed that this latter model best fitted the sequence data $(\mathrm{LRT}=23.86, \mathrm{df}=2, \mathrm{p}=0.001$, Table 1$)$. To determine if this result was a consequence of positive selection or relaxed evolutionary constraints, a comparison was made between the likelihood values of the MA model with the $\mathrm{MA}_{\omega=1}$ model. This test indicates that the branch corresponding to the first duplication displays evidence of positive selection (LRT $=12.68, \mathrm{df}=2$, $\mathrm{p}=0.005$ ). Four amino acid residues were identified as being under positive selection with BEB (Bayes Empirical Bayes) values greater than 0.95 (Table 1). Branch $b$ and $c$ exhibited a $d_{\mathrm{N}} / d_{\mathrm{S}}$ ratio $(\omega)$ value close to infinity (Fig. 1a) but, when tested for positive selection signatures, MA did not better fit the data than M1a (Table 1). This was mainly due to the fact that the two internal branches contained

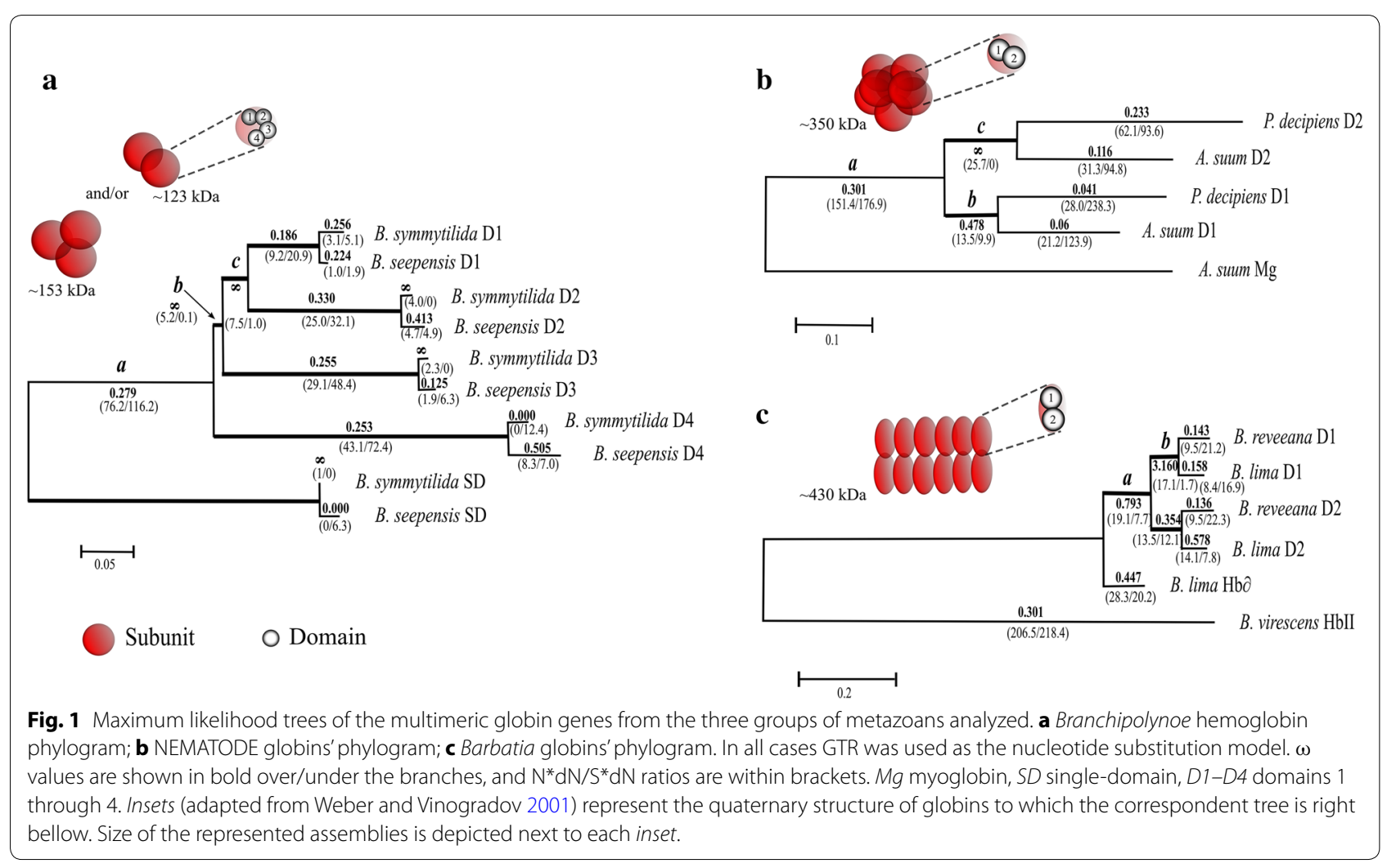


Table 1 Results from the analyses of positive selection (PAML v 4.7, Yang 2007)

\begin{tabular}{|c|c|c|c|c|c|c|}
\hline Model & $\ln L$ & к & $\mathrm{np}$ & Model estimates & LRT (df) & $\begin{array}{l}\text { Sites under positive } \\
\text { selection }(B E B>0.95)\end{array}$ \\
\hline \multicolumn{7}{|l|}{ Branchipolynoe } \\
\hline \multicolumn{7}{|l|}{ Branch model } \\
\hline Mo & $-2,061.60$ & 1.755 & 19 & $\omega=0.278$ & & \\
\hline M1 & $-2,052.07$ & 1.781 & 35 & $0.001<\omega<\infty$ & $19.05^{\mathrm{NS}}(16)$ & \\
\hline \multicolumn{7}{|l|}{ Site model } \\
\hline M1a'nearly neutral' & $-2,050.01$ & 1.842 & 20 & $\begin{array}{l}\omega_{0}=0.177(79 \%) \\
\omega_{1}=1.000(21 \%)\end{array}$ & & \\
\hline M2a'positive selection' & $-2,050.01$ & 1.842 & 22 & $\begin{array}{l}\omega_{0}=0.177(79 \%) \\
\omega_{1}=1.000(10.8 \%) \\
\omega_{2}=1.000(10.2 \%)\end{array}$ & $0.00^{\mathrm{NS}}(2)$ & \\
\hline \multicolumn{7}{|l|}{ Branch-site model } \\
\hline MA branch a (duplication) & $-2,038.08$ & 1.851 & 22 & $\begin{array}{l}\omega_{0}=0.154(65.6 \%) \\
\omega_{1}=1.000(16.3 \%) \\
\omega_{2 a}=\infty(14.5 \%) \\
\omega_{2 b}=\infty(3.6 \%)\end{array}$ & $23.86^{* * * * *}(2)$ & $\begin{array}{l}27 V \\
30 A \\
52 Q \\
65 C\end{array}$ \\
\hline MA branch b (D4 vs D3 D2 D1) & $-2,049.37$ & 1.845 & 22 & $\begin{array}{l}\omega_{0}=0.167(0 \%) \\
\omega_{1}=1.000(0 \%) \\
\omega_{2 a}=1.076(78.5 \%) \\
\omega_{2 b}=1.076(21.5 \%)\end{array}$ & $1.29^{\mathrm{NS}}$ & - \\
\hline MA_branch c (D2 D1) & $-2,048.87$ & 1.856 & 22 & $\begin{array}{l}\omega_{0}=0.167(0 \%) \\
\omega_{1}=1.000(0 \%) \\
\omega_{2 a}=2.6(79.6 \%) \\
\omega_{2 b}=2.6(20.4 \%)\end{array}$ & $2.28^{\mathrm{NS}}$ & - \\
\hline \multicolumn{7}{|l|}{ Nematodes } \\
\hline \multicolumn{7}{|l|}{ Branch model } \\
\hline Mo & $-2,153.41$ & 1.455 & 9 & $\omega=0.037$ & & \\
\hline M1 & -2146.71 & 1.313 & 15 & $0.029<\omega<\infty$ & $13.4^{*}(6)$ & \\
\hline \multicolumn{7}{|l|}{ Site model } \\
\hline M1a'nearly neutral' & $-2,141.70$ & 1.476 & 10 & $\begin{array}{l}\omega_{0}=0.071(87.3 \%) \\
\omega_{1}=1.000(12.7 \%)\end{array}$ & & \\
\hline M2a'positive selection' & $-2,141.70$ & 1.476 & 12 & $\begin{array}{l}\omega_{0}=0.071(87.3 \%) \\
\omega_{1}=1.000(3.0 \%) \\
\omega_{2}=1.000(9.7 \%)\end{array}$ & $0^{\text {NS }}$ & \\
\hline \multicolumn{7}{|l|}{ Branch-site model } \\
\hline MA branch a (duplication) & $-2,133.19$ & 1.565 & 12 & $\begin{array}{l}\omega_{0}=0.074(51.2 \%) \\
\omega_{1}=1.000(7.2 \%) \\
\omega_{2 a}=1.412(36.4 \%) \\
\omega_{2 b}=1.412(5.2 \%)\end{array}$ & $\begin{array}{l}17.02^{* * * * *}(2) \\
\text { MA vs MA }{ }_{\omega=1}^{\text {NS }}\end{array}$ & 16 sites \\
\hline MA branch $b$ (D1) & $-2,135.16$ & 1.407 & 12 & $\begin{array}{l}\omega_{0}=0.063(74.2 \%) \\
\omega_{1}=1.000(10.2 \%) \\
\omega_{2 a}=\infty(13.7 \%) \\
\omega_{2 b}=\infty(1.9 \%)\end{array}$ & $13.09^{* * * *}(2)$ & $11 \mathrm{~A}$ \\
\hline MA branch c (D2) & $-2,128.13$ & 1.373 & 12 & $\begin{array}{l}\omega_{0}=0.061(79.1 \%) \\
\omega_{1}=1.000(9.5 \%) \\
\omega_{2 a}=\infty(10.2 \%) \\
\omega_{2 b}=\infty(1.2 \%)\end{array}$ & $27.14^{* * * * *}(2)$ & - \\
\hline \multicolumn{7}{|l|}{ Barbatia } \\
\hline \multicolumn{7}{|l|}{ Branch model } \\
\hline Mo & $-1,989.05$ & 1.398 & 11 & $\omega=0.332$ & & \\
\hline M1 & $-1,980.29$ & 1.393 & 19 & $0.136<\omega<3.160$ & $17.54^{* *}(8)$ & \\
\hline \multicolumn{7}{|l|}{ Site model } \\
\hline M1a & $-1,967.14$ & 1.514 & 12 & $\begin{array}{l}\omega_{0}=0.128(62 \%) \\
\omega_{1}=1.000(34 \%)\end{array}$ & & \\
\hline $\mathrm{M} 2 \mathrm{a}$ & $-1,956.20$ & 1.649 & 14 & $\begin{array}{l}\omega_{0}=0.120(54.9 \%) \\
\omega_{1}=1.000(43.1 \%) \\
\omega_{2}=\infty(2 \%)\end{array}$ & $21.88^{* * * * *}(2)$ & \\
\hline
\end{tabular}


Table 1 continued

\begin{tabular}{|c|c|c|c|c|c|c|}
\hline Model & $\ln L$ & $\kappa$ & $\mathrm{np}$ & Model estimates & LRT (df) & $\begin{array}{l}\text { Sites under positive } \\
\text { selection }(B E B>0.95)\end{array}$ \\
\hline \multicolumn{7}{|l|}{ Branch-site model } \\
\hline MA branch a (duplication) & $-1,961.52$ & 1.572 & 14 & $\begin{array}{l}\omega_{0}=0.119(59.9 \%) \\
\omega_{1}=1.000(29.5 \%) \\
\omega_{2 a}=\infty(7.1 \%) \\
\omega_{2 b}=\infty(3.5 \%)\end{array}$ & $11.24^{* * *}(2)$ & - \\
\hline MA branch $b$ (D1) & $-1,962.64$ & 1.510 & 14 & $\begin{array}{l}\omega_{0}=0.117(62.3 \%) \\
\omega_{1}=1.000(31.5 \%) \\
\omega_{2 a}=\infty(4.1 \%) \\
\omega_{2 b}=\infty(2.1 \%)\end{array}$ & $9.00 * *(2)$ & $31 \mathrm{G}$ \\
\hline
\end{tabular}

In the 4 invertebrate groups, the likelihood (In) values correspond to branches leading to various duplications (see Fig. 1a-c) in each phylogram. Selection models implemented in the codeml package, its parameters and associated results of likelihood ratio tests (LRT) are shown (significance threshold: $\mathrm{p}<0.05$ ).

$\kappa$ transition/transversion ratio, $n p$ number of parameters estimated by the model, model estimates: $\omega_{0}$ estimated $\omega$ for the category of sites under purifying selection $(\omega<1), \omega_{1}$ estimated $\omega$ for sites for the category under neutral evolution $(\omega \sim 1), \omega_{2 a}$ estimated $\omega$ for sites under positive selection in the foreground branches against background branches under purifying selection, $\omega_{2 b}$ estimated $\omega$ for sites under positive selection in the foreground branches against background branches under neutral evolution, $d f$ degrees of freedom, NS non significant.

* Significant at $0.05 ;{ }^{* *}$ significant at $0.025 ;{ }^{* * *}$ significant at $0.01 ;{ }^{* * *}$ significant at $0.005 ;{ }^{* * * *}$ significant at $0.001 ; \mathrm{BEB}>0.95$ : sites identified by Bayes Empirical Bayes analysis with a posterior probability greater than $95 \%$.

very few mutations, most of them being nonsynonymous mutations ( 6 and 5 replacements, respectively), and no position had a BEB probability $>95 \%$.

All other internal branches had quite relaxed $\omega$ values between 0.255 and 0.33 . They were all tested but no signature of positive selection was found. The $\omega$ values for the terminal branches were generally characteristic of purifying selection $(\omega \ll 1)$. Interestingly, the exceptions were all on branches leading to B. symmytilida sequences (SD, D3, and D2). The results of LRT for these 3 branches showed that M1a model (almost neutral) was systematically chosen against MA with one to two sites positioned in the $\omega>1$ class. Although not significant (BEB probability ranged between 0.51 and 0.74 ), these replacements were all found within B. symmytilida, suggesting that this newly formed species might have encountered changing conditions since speciation.

Although REL (Random Effects Likelihood, episodic diversifying selection) analysis identified branch $a$ as exhibiting the strongest signs of positive selection, the corrected $p$ value was not significant $(\mathrm{p}=0.13)$, and the sites identified as under positive selection with PAML were not supported by the MEME analysis.

\section{Di-domain nematode Hbs: positive selection in one of the two domains}

Based on earlier work (Blaxter 1993; Blaxter et al. 1994; Hunt et al. 2009) we chose the single-domain globins sequences from $A$. suum to serve as outgroup in our tests for selective regimes. In agreement with earlier work and our alignment (Additional file 1: Figure S1) with the sequences of the hemoglobins from $A$. suum and $P$. decipiens, the orthologous sequences of each domain indicated that the duplication occurred before the separation of the two species. We used this unrooted topology (Fig. 1b) for our analysis of selective pressures during the evolution of these di-domain globins.

The 'free-ratio' model better fits the sequence data than the 'one-ratio' model (LRT $=15.38, \mathrm{df}=6, \mathrm{p}=0.05$ ), indicating that the $d_{\mathrm{N}} / d_{\mathrm{S}}$ ratios $(\omega)$ are heterogeneous among branches. In the branch leading to the nematode multi-domain hemoglobins (branch $a$, Fig. 1b), $\omega$ was 0.301 , with numerous synonymous and non-synonymous substitutions. For this branch, the 'site model' test indicated that the MA model had a significantly greater likelihood value than the M1a model $(\mathrm{LRT}=17.02, \mathrm{df}=2$, $\mathrm{p}=0.001$ ), and BEB analyses identified 16 sites under positive selection with a probability greater than $95 \%$. However, the likelihood for MA was not significantly different from that for $\mathrm{MA}_{\omega=1}(\mathrm{LRT}=0.29, \mathrm{df}=2)$, indicating that there was no positive selection in the branch leading to the multi-domain nematode $\mathrm{Hb}$ or that it was masked by saturation of the phylogenetic signal (accumulation of a very large number of synonymous mutations).

On the branches corresponding to the duplicated domains (branch $b$ for domains D1, and branch $c$ for domains D2; Fig. 1b), there are more non-synonymous mutations per non-synonymous site than synonymous mutations per synonymous sites, consistent with the expectations for positive selection. For both branches, MA (branch-site model) better fits the data than M1a (Table 1), indicating heterogeneity of selective pressures among sites. For both of these branches, the LRT between $\mathrm{MA}$ and $\mathrm{MA}_{\omega=1}$ was significant (branch $b$ : $\mathrm{LRT}=8.31, \mathrm{df}=2, \mathrm{p}=0.025$; branch $c: \mathrm{LRT}=11.51$, $\mathrm{df}=2, \mathrm{p}=0.005)$, more specifically indicating the action of positive selection for some amino acid positions. The Bayesian analysis however did not reveal any amino acid 
position as being significantly under positive selection for domain D2 and only the alanine residue at position 11 was positively selected for D1 (Table 1).

Again, the search for episodic diversifying selection (REL analysis) did highlight branch D1 as well, but the corrected value is not significant, and according to this method the amino acid alanine in position 11 does not appear to be under positive selection.

\section{Di-domain intracellular blood clam Hbs}

The $\delta$ globin chain is considered to be the ancestral single-domain globin sequence for the di-domain of $B$. lima (Suzuki et al. 1996). Our determination of selective forces that acted during the di-domain globin gene evolution was performed with this globin sequence as the outgroup for the phylogeny (Fig. 1c).

The comparison between the 'one-ratio' model and the 'free-ratio' model indicates that $\omega$ is not homogenous among branches ( $\mathrm{M} 1$ vs. $\mathrm{M} 0, \mathrm{LRT}=17.54 \mathrm{df}=8$, $\mathrm{p}=0.025)$. The MA model best fits the data than M1a for branches $a$ and $b$ (branch-site model; Table 1), suggesting that some amino acids could be under positive selection in these branches. The BEB analysis revealed that residue $31 \mathrm{G}$ (located in the $\mathrm{AB}$ corner) was under positive selection. None of the other branches exhibited amino acid positions with signature of positive selection.

The REL analysis did not support the findings of the PAML analysis in the blood clam Hbs.

\section{Location of the amino acid sites under positive selection in a 3D model}

The 3D modeling was only performed for the datasets in which we detected potential amino acids under positive selection and a 3D model of the globin subunit was created for only one of the species inside each group of invertebrates.

\section{Annelids}

The similarity of the four domains, between the 2 species of Branchipolynoe ranged between 94 and 97\% (Additional file 2: Table S1). Based on this high level of similarity, we only produced the 3D homology model of domain 1 from B. symmytilida (Fig. 2a). Three templates were used to produce the 3D models of the Hb D1 from B. symmytilida. The first one was the $\mathrm{Hb}$ from the polychaete Glycera dibranchiata [Protein Data Bank (PDB) $1 \mathrm{HBG}$ ] as this is one of the closest species with a globin crystal. The second corresponds to another annelid species, Lumbricus terrestris (PDB 1X9F), and the third template was $\mathrm{Hb}$ from the cestode Gasterophilus intestinalis (PDB 2COK), the closest sequence automatically chosen by the SWISS-MODEL server. Out of the three models, the one that we considered the best was the model based on the Lumbricus $\mathrm{Hb}$ sequence (based on the ANOLEA and GROMOS graphics, and by comparing the QMEAN descriptors-see details in "Methods"). Although the model based on G. intestinalis had a smaller QMEAN value (the lower the predicted energy, the better the model) the Lumbricus-based model exhibited smaller errors in the parts of the quaternary structure where the residues under positive selection are located.

The cysteine in position $65(65 \mathrm{C})$ is located in the far end of the heme pocket and is surrounded by a slightly hydrophilic and polar cluster (Additional file 3: Figure S2). The remaining amino acids identified by BEB analysis under positive selection are located in the $B$ helix (27V and 30A), close to the DE corner, and on the E helix (52Q) (Fig. 2a). The substitutions of histidine for a valine and glutamine for an alanine on the B helix from the single-domain to the tetra-domain produce a more hydrophobic exposed surface of the protein in the area. In the vicinity of the $\mathrm{DE}$ corner, the replacement of an alanine by a glutamine in position 52 (E5), on the contrary, creates a more hydrophilic surface. When using $L$. terrestris crystal structure to model the theoretical tetramer based on B. symmytilida D1 sequence, it becomes clear that this same residue $(52 \mathrm{Q})$ occupies a central position in the tetramer (Fig. 3).

\section{Nematodes}

A crystal structure was already available in the PDB for Ascaris hemoglobin D1 (1ASH), and this was the chosen template to model D2 (SWISS-MODEL server). We identified a single residue in domain D1 as being under positive selection (A11) through BEB analysis. This amino acid is located on the loop between helices $\mathrm{A}$ and B (Fig. 2c), and points toward the core of the protein, suggesting that the substitution could affect the stability of helix A. This Ala replaces an Ile and, even though both are hydrophobic, the latter is larger and strongly hydrophobic. The Ile forms closer interactions with other hydrophobic amino acids, in particular on helix E (Leu in position 66 for D2 and 69 in D1) and, to a lesser extent, with the end of helix G (Leu in position 119 on D1 and 116 in D2; Fig. 2c). Ile13 (in D2) and Val16 (in D1) are also likely to participate in this hydrophobic cluster. The Ala in position 11 could allow a looser interaction with the hydrophobic cluster located in this region, possibly resulting in a more flexible region of the protein.

\section{Globin domain of blood clams}

The similarity between each hemoglobin domain of $B$. reeveana and B. lima was $\sim 91 \%$ for domain 1 and $\sim 88 \%$ for domain 2 (Additional file 2: Table S1). The high level of identity allowed us to only use one of the two domains from one species to produce the 3D model. The model 

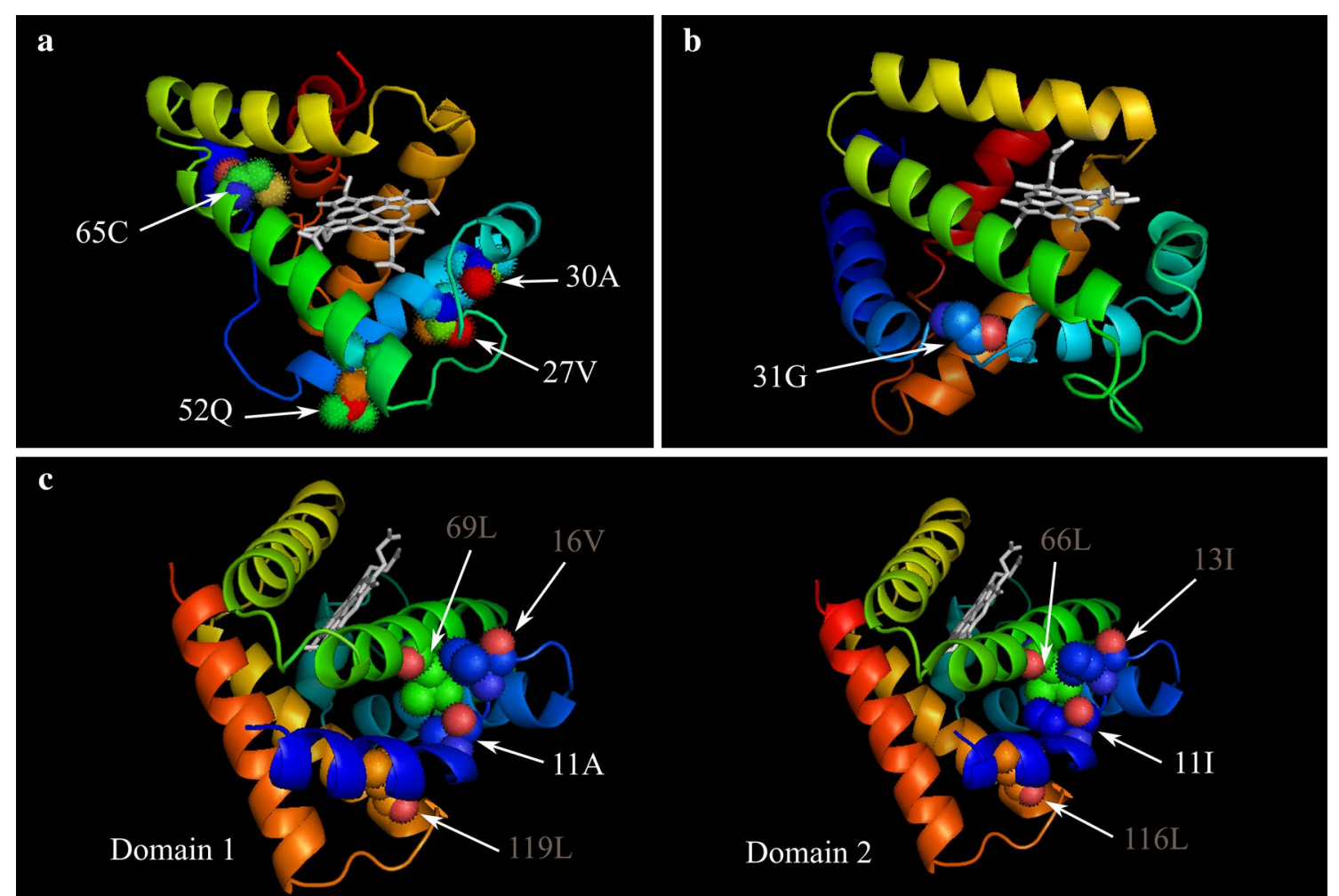

Fig. 2 3D models of the globins found in the different species studied, with their heme group. a The B. symmytilida model was obtained based on L. terrestris $\mathrm{Hb}$ structure; residues under positive selection are labeled in white (27V, 30A, 52Q and 65C). b The B. reveeana model is based on the tertiary structure of $\mathrm{S}$. inaequivalis $\mathrm{Hb}$; the residue under positive selection is shown (31G). c Ascaris D2 model was built based on the di-domain, D1 crystal (1ash); residue under positive selection on D1 is depicted (11A), name of the residues with possible interactions with amino acid in position 11 are shown in grey. A comparative scenario is presented for Ascaris D1 and D2 with the interacting residues being in equivalent positions in the protein tertiary structure. Helices are represented in a color spectrum depicting helix $\mathrm{A}$ in blue all through helix $\mathrm{H}$ in red. Depicted residues are colored by element; $\mathrm{OH}$ : red; NH: blue: C: helix color. Heme group is shown in light grey. See "Methods" and "Results" sections for detailed information.

of $\mathrm{D} 1$ from $B$. reeveana $\mathrm{Hb}$ was automatically obtained through the homology of the quaternary structure of the hemoglobin from Scapharca inaequivalvis (PDB 1SCT), the closest sequence with a crystal structure currently available. S. inaequivalis is a bivalve from the family Arcidae, specialized in anoxic and sulfidic environments (de Zwaan et al. 1995). The packing quality of the model (ANOLEA) and the empirical force field (GROMOS) were overall good (more negative energy values), including the area were we found the residue under positive selection. The residue 31G, identified as being under positive selection by BEB analysis, is located in the corner between helices A and B (Fig. 2b). This uncharged weak polar residue is surrounded by a strong hydrophilic polar cluster (Additional file 4: Figure S3).

\section{Discussion}

The basic tertiary structure of globins-the globin fold-is highly conserved in all domains of life (Weber and Vinogradov 2001). Although vertebrate circulating hemoglobins always exhibit a tetrameric structure involving two types of subunits (the iconic $\alpha_{2} \beta_{2}$ structure), invertebrate hemoglobins have a great diversity of quaternary structures, from dimers to a staggering 180-subunit assemblage (Weber and Vinogradov 2001; Royer et al. 2005). Despite this great structural diversity, all invertebrate allosteric hemoglobins possess a similar basic dimeric unit, termed "EF dimers" because of the extensive implication of amino acid residues from helices $\mathrm{E}$ and $\mathrm{F}$ at the interface between the two subunits (Royer et al. 2001). Further assembly of subunits is obtained by interaction of these basic EF dimers. The high level of conservation of the basic globin folding and inter-subunit interactions allows us to make predictions about the role that the amino acids under positive selection could play. This study of three very diverse taxonomic lineages allowed us to test whether the predicted structural constraints produced similar selective response (adaptive 


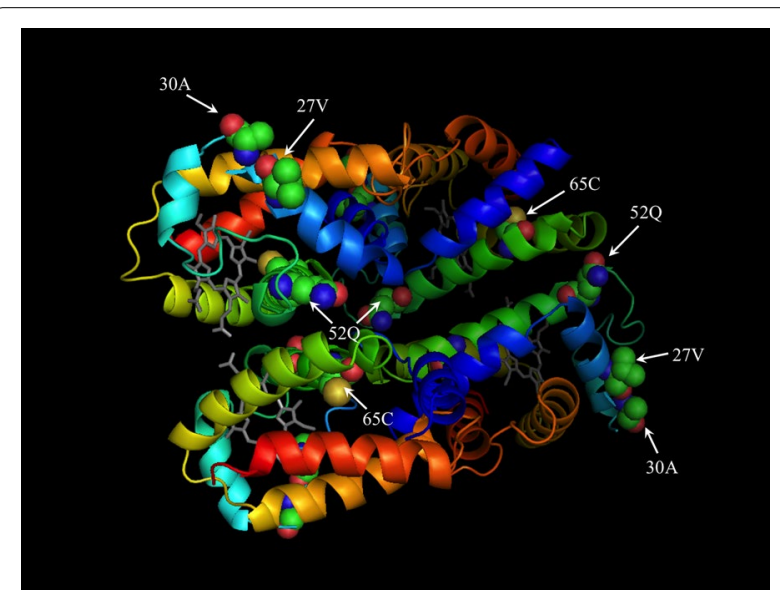

Fig. 3 Theoretical 3D model of an annelid tetramer based on B. symmytilida D1 sequence and the crystal structure of Lumbricus terrestris (1X9F). Amino acids identified as potentially under positive selection are highlighted. 52Q appears to be in an area where it could participate to the formation of an oligomer. Depicted residues are colored by element; $\mathrm{OH}$ : red; $\mathrm{NH}$ : blue: $\mathrm{C}$ : helix color. Heme group is shown in grey. Model constructed with Modeller (Mod9v13, Eswar et al. 2006).

structural convergence) when facing challenging environmental conditions (in this case hypoxia). Positive selection could also affect the function of each domain differentially, leading to domains with different, specialized characteristics (neofunctionalization).

In all our analyses, the findings of the PAML analyses were not significantly supported by the REL analyses, although the branches identified by the former were also the ones exhibiting the strongest signal of episodic diversifying selection in the latter type of analyses. The following discussion is therefore centered on the PAML results. Although care should be taken when using $\mathrm{d}_{\mathrm{N}} / \mathrm{d}_{\mathrm{S}}$ analyses and BEB determination of amino acids under positive selection (Zhang et al. 2005), the identified residues occupy positions for which structural and/or functional predictions can be made (inter- and intra-subunit interactions, affinity for oxygen).

\section{Annelida: a recent and special multi-domain $\mathrm{Hb}$}

Multi-domains $\mathrm{Hbs}$ in annelids are so far only known in species of the genus Branchipolynoe, that lives commensally with mussels from hydrothermal vents and cold seeps (Hourdez et al. 1999a). These hemoglobins evolved recently (ca. $60 \mathrm{Ma}$ ago) in this group of scaleworms, and were obtained by tandem duplication of a single-domain gene coding for a myoglobin-like protein (Projecto-Garcia et al. 2010). We detected that diversifying selection has impacted the primary structure of the Branchipolynoe Hbs domains before the duplication process, and this $d_{\mathrm{N}} / d_{\mathrm{S}}$ heterogeneity was due to positive selection that acted in the branch corresponding to the initial duplication event. This suggests that amino acid changes were important for the formation of the multi-domain globin, for structural and/or functional reasons. The other internal branches of the domains' phylogeny all had $\omega \ll 1$, suggesting that after the positive selection at the first duplication event, purifying selection acted to conserve the modified amino acids, and that no further modification was necessary to allow the functioning of the globin with more than two domains. Some of the terminal branches for B. symmytilida also display high $\omega$ values. This could indicate specialization of this species tetra-domain $\mathrm{Hb}$ to slightly different environmental conditions. In the evolution of the Branchipolynoe species the Pacific $B$. symmytilida, living around vents, seems to derive from a cold seep ancestor, morphologically close to the Atlantic B. seepensis, whose actual 'cold seep' intermediates are presently located on both parts of the Panama Isthmus. Alternately, there are two types of hemoglobins in Branchipolynoe, one forms dimers and the second trimers (Hourdez et al. 1999a). It is possible the sequences used here are paralogs rather than orthologs. If this is the case, our results could be indicative of subfunctionalization after tetra-domain gene duplication. The BEB test did not detect specific amino acids under positive selection on the terminal branches. However, the branch-site model and BEB analysis are probably not powerful enough when the number of nucleotide substitutions in the foreground branch is small (Nozawa et al. 2009).

Duplication events are common in eukaryote lineages, producing gene families, with paralogs either carrying on the function of the parent gene or giving rise to novel functions (by neofunctionalization or subfunctionalization; Britten and Kohne 1968; Duboule and Wilkins 1998; Voordeckers et al. 2012). Assuming an intracellular globin gene as the parental gene for the tetra-domain lineage (Projecto-Garcia et al. 2010), the paralogs seem to have functions similar to that of their ancestor. Branchipolynoe tetra-domain globins possess high oxygen affinity and a low cooperativity (Hourdez et al. 1999b), both characteristics reminiscent of myoglobin function, suggesting that the amino acid changes affect the structure rather than the function of the multi-domain hemoglobin. Branchipolynoe extracellular hemoglobins also form multimers (Hourdez et al. 1999a) while myoglobins are usually monomeric. The observed changes could then be related to this capacity to form oligomers.

Some of the amino acids under positive selection are located in the B helix (27 V and 30A), close to the DE corner (Fig. 2a). Because of their position and their hydrophobicity these amino acids are likely to be involved in the interface between two domains. In multi-domain 
proteins the residues found in interface regions are mostly non-polar to allow stable interaction regions (Bhaskara and Srinivasan 2011) and in both positions, 27 and 30, polar residues were substituted by non-polar derived amino acids, $\mathrm{H} \rightarrow \mathrm{V}$ and $\mathrm{Q} \rightarrow \mathrm{A}$, respectively (Additional file 3: Figure S2). The glutamine in position $52(52 \mathrm{Q})$ is in the E helix, and could be involved in the formation of oligomers (Fig. 3), which usually involves the interaction of the helices $\mathrm{E}$ and $\mathrm{F}$ between subunits (Royer et al. 2005). In this position the ancestral nonpolar residue, an alanine, was substituted by a glutamine, a polar amino acid. This could be advantageous as, based on the probable configuration of the multi-domain folding (Fig. 3), this glutamine can establish a hydrogen bond with the polar serine (located early in the F helix). The $65 \mathrm{C}$ is located in the far end of the heme pocket, but the distance to the distal heme is too large to affect oxygenbinding properties. This cysteine is particularly interesting as it is under positive selection in some extracellular globins from other annelids that live in sulfidic environments (Bailly et al. 2003). This amino acid, located at the exact same position, was thought to be involved in the reversible binding of sulfide in Riftia (Zal et al. 1998) but this mechanism of sulfide binding has since been challenged (Flores et al. 2005; Flores and Hourdez 2006). Its occurrence in a lineage of extracellular globins distinct from the typical HBL-Hb globins (and its absence in the single-domain intracellular globins) suggests that it is the result of convergent evolution. Its function remains unclear but its presence in an extracellular hemoglobin from a species that lives under sulfidic conditions points towards a possible function in protecting the heme from reacting with sulfide (which would form sulfhemoglobin, no longer capable of reversibly binding oxygen).

\section{Nematode $\mathrm{Hb}$}

Nematode globins have a very rich evolutionary history (Blaxter 1993; Hoogewijs et al. 2008; Hunt et al. 2009). The duplication of this multi-domain $\mathrm{Hb}$ gene most likely happened in the common ancestor of Ascaris and Pseudoterranova, because each domain clusters together (Blaxter 1993). In both species the di-domain Hbs exhibit a high oxygen affinity, and share amino acid residues in the heme pocket that are thought to be responsible for the slow release of oxygen from the heme (Gibson et al. 1993).

These are the highest oxygen affinities measured in animal globins, and we therefore expected to find signatures of positive selection in the lineage leading to the duplicated nematode $\mathrm{Hb}$ gene. The fact that we did not find positive selection in the branch between the single- and the di-domain globins could be due to two reasons: (1) the time of the duplication (by unequal crossing-over) is estimated to be $245 \mathrm{Ma}$ in P. decipiens (Dixon et al.1992) (and we can assume the same time for A. suum $\mathrm{Hb}$ gene duplication based on their orthology), a time probably sufficient to erase signatures of selection because of the accumulation of synonymous substitutions (Yang 2008); and (2) the fact that the residues (B10Y and E7Q) that are thought to be responsible for the high oxygen affinity in the di-domains (De Baere et al. 1994) are also present in the myoglobin of A. suum (Blaxter et al. 1994), used in these study as an outgroup for our topology. However, Blaxter et al. (1994) suggest that these residues may not be the only requirement for high oxygen affinity, other amino acids possibly affecting the distance between the B10Y residue and the di-oxygen bound to the heme might be also responsible.

As in annelids, it seems that the amino acid we identified as being under positive selection more likely has a structural rather than functional effect. The residue $11 \mathrm{~A}$ could be responsible for a greater flexibility of the protein, in particular by affecting the stability of the E-helix. This amino acid could indeed form weaker hydrophobic bonds with residues on the $\mathrm{A}-\mathrm{B}$ corner and the E-helix. This could also be important during $\mathrm{O}_{2}$ association/dissociation processes and, according to Kloek (1993), D1 does exhibit a faster $\mathrm{O}_{2}$ dissociation rate than D2.

It is possible that the most important difference between a single-domain and a di-domain $\mathrm{Hb}$, in Ascaris, is the fact that the di-domain $\mathrm{Hb}$ can form octamers (Darawshe et al. 1987; De Baere et al. 1992). In Ascaris lumbricoides, the octameric structure is obtained by the interaction of a highly charged $\mathrm{C}$-terminus tail that acts as an intra-molecular chaperone, and the interaction is then stabilized by interactions between globin folds, especially by the leucine residue in position 15 (Minning and Goldberg 1998).

\section{Molluskan Hbs}

Mollusks have remarkable multi-domain Hbs. Not only are their genes made of more that one domain, the number of repeats of these domains is of a magnitude only found in other protein families in vertebrates (Björklund et al. 2005). The di-domain Hb from Barbatia did not reach a spectacular state of duplication, it is, however, an intracellular $\mathrm{Hb}$, and in this category its molecular weight ( $\sim 30 \mathrm{kDa}$, result of $\sim 35 \mathrm{kDa}$ subunits assemblage in dodecamers) remains unrivaled (Grinich and Terwilliger 1980; Grinich et al. 1986). The D1 lineage from Barbatia exhibits a $\omega$ greater than 1, in sharp contrast with the D2 lineage with an $\omega \sim 0$, highlighting structural (and possibly functional) changes on D1, while D2 experiences slight purifying selection $(\omega=0.354)$. The residue $31 \mathrm{G}$ (AB corner) was identified as being under positive selection in the 
D1 lineage. This corner, in particular, is extensively involved in contacts used to assemble the dimers of HbII into tetramers in Scapharca, another arcid clam (Royer et al. 1995). In Barbatia, it is mainly a hydrophilic region (Additional file 4: Figure S3): the residue occupying this position is a glycine (weakly polar) in D1 and a lysine (polar) in D2. The very different size of the side chains is likely to affect the oligomerization of these subunits, and glycine would probably have a stabilizing effect in this interaction region through its lower hydrophilic character. Lysine in this position is found not only in the D2 of B. lima and B. reveeana, but also with the outgroup sequences, $\mathrm{Hb} \delta$ from $B$. lima and HbII from B. virescens, supporting its importance in comparison with the same site in D1. The presence of a short interdomain peptide (termed 'linker') in Barbatia (Naito et al. 1991) may also allow a greater flexibility between the two domains, and thereby relax the pressure that the too-close setting of the tandem duplication represents. Only the crystallization of the native $430 \mathrm{kDa}$ component of Barbatia hemoglobin or its $220 \mathrm{kDa}$ dissociation product (Grinich and Terwilliger 1980) could shed light of the amino acids involved in actual interactions, and the potential effect of the linker.

\section{Conclusion}

\section{Multi-domain proteins: selection and folding}

Our analyses on all three datasets of multi-domain proteins showed that most amino acid positions are under moderate purifying selection $(\omega<1)$, some appear to behave neutrally $(\omega=1)$, and very few have been under positive selection. These latter however seem to be mainly located in positions where they can affect the structure rather than the function of the proteins.

Based on genome comparisons (from prokaryotes and eukaryotes), it seems that the occurrence of a single duplication event is much more common (giving rise to di-domains) in protein genes than several duplication events (Apic et al. 2001; Björklund et al. 2005), except for protein genes that were already multi-domain. In this case the replication of several domains at once is usually the norm, producing domain repeats (Björklund et al. 2005). As for other metazoans, the domain duplication in Branchipolynoe could be an internal response to a specific environmental constraint (Apic et al. 2001). In Branchipolynoe's case, the low levels of oxygen (hypoxia) that result from the mixing of the anoxic hydrothermal vent fluid with the deep-sea water could be the environmental constraint that favored the appearance of the multi-domain hemoglobin (Hourdez et al. 1999b). Hypoxia may also be the constraint responsible for the multi-domain hemoglobin evolution for the two other taxa: the two nematodes are gut parasites of vertebrates, and the bivalve is found in low oxygen marine environments.

All the discussed multi-domain Hbs have different evolutionary single-domain points of origin, the first duplication occurred at very different times: estimated to be about $245 \mathrm{Ma}$ ago for the nematodes (Dixon et al. 1992), before the speciation process that led to the emergence of Barbatia species about $165 \mathrm{Ma}$ ago (Plazzi and Passamonti 2010, and less than $60 \mathrm{Ma}$ ago for Branchipolynoe, as the duplication event occurred after the scale-worm radiation (Projecto-Garcia et al. 2010). This will undoubtedly have a profound blurring effect on the signal of selective pressures in the deeper branches of the gene phylogenies, as more synonymous substitutions will have accumulated in the older lineages and the positive selection signal attenuated.

The proper folding of multi-domain proteins (and in particular tandem duplicated ones) can be problematic if the contact areas are not sufficiently different (Han et al. 2007). As a result, contact areas in multi-domain proteins usually consist of less similar domains (Han et al. 2007). The residues in Branchipolynoe $\mathrm{Hb}$, near the $\mathrm{DE}$ corner, in the $B$ and $E$ helices are serious candidates to achieve a proper folding of the tetra-domain $\mathrm{Hb}$, as well as polymerization to form the dimers and trimers of tetra-domain globins found in Branchipolynoe (Hourdez et al. 1999a). To date, there are no known interactions of these Hbs with other proteins and the observed changes are unlikely to correspond to intermolecular coevolution. However, this possibility cannot be ignored for other multi-domain proteins.

In invertebrate multi-domain globins, the polymerization and cooperativity depend mostly on the E-F dimer structure (interactions between $\mathrm{E}$ and $\mathrm{F}$ helices from different subunits) (Royer et al. 2001; Riggs 1998). Bigger structures, opposed to a myoglobin or single-domain $\mathrm{Hb}$, may be important to avoid their accidental elimination or to maintain a low oncotic pressure while increasing oxygen carrying capacity.

\section{Support of predictive models of interdomain interactions}

These observations raise the interesting possibility that this approach could be used as a method that points towards amino acids found at the interface between two domains of a protein. In particular, it could be used in support of predictive models for intermolecular contacts. It is indeed sometimes difficult to obtain crystals of multidomain proteins, and research must rely on isolated domains structures. The approach we used could be used in virtually all mutidomain proteins, and help identify interaction areas if these are not known from crystal structures. 


\section{Methods}

\section{Sequence retrieval}

Multi-domain globin sequences for mollusks, annelids, and nematodes were retrieved from Genbank. The selection criteria for the $\mathrm{Hb}$ sequences were to find a phylogenetic group where one could find a multi-domain $\mathrm{Hb}$, and that has a single-domain globin (or a closely related taxon with this single-domain globin) for comparison. For arthropods, as well as for some mollusks, this was not possible. As a result, we only used globin sequences from Barbatia reveeana (M73328), B. lima (delta chain $\mathrm{Hb}$ : D63932, alpha chain Hb: D63933, beta chain Hb: D63934, di-domain $\mathrm{Hb}$ : D58417), B. virescens (chain II $\mathrm{Hb}$ : D58416). Although sequences for the snail Biomphalaria glabrata are available, a reconstruction of the phylogeny of the 13 globin domains yielded very low confidence values for most of the deep branches (data not shown). This could greatly affect our confidence in the reconstruction of ancestral sequences, and this dataset was therefore cast aside. For nematodes: we retrieved sequences from Ascaris suum (myoglobin: U17337, di-domain $\mathrm{Hb}$ : L03351), Pseudoterranova decipiens (di-domain $\mathrm{Hb}$ : M63298) and the single-domain globins from Caenorhabditis elegans (Z18264) and Trichostrongylus colubriformis (M63263). For annelids we used sequences from two species of the scale-worm genus Branchipolynoe; $B$. symmytilida and B. seepensis (tetra-domain globins GQ360749-GQ360756, and their single-domain globins GQ360757-GQ360758, respectively).

\section{Phylogenetic analyses}

For all sequence datasets, multiple nucleotide and amino acid sequence alignments were performed with the multiple sequence alignment algorithm MUSCLE (Edgar 2004, part of software Geneious Pro 5.3.6, created by Biomatters). During sequence alignment optimization, we aimed at minimizing the number of indels, and because we were dealing with coding sequences, the nucleotide alignment was constrained by the amino acid sequences alignment. Although the number of sequences was small in each taxonomic group, we compared the alignments obtained with MUSCLE and submitted the raw amino acid sequences to the GUIDANCE filter (Penn et al. 2010), using the alignment algorithm MAFFT (Katoh et al. 2005). MUSCLE and MAFFT produced very similar outputs and we therefore chose the alignments produced by the former (Additional file 1: Figure S1, Additional file 3: Figure S2, Additional file 4: Figure S3). GUIDANCE provided us alignment scores and regions of the alignments that were not well supported (Additional file 1: Figure S1, Additional file 3: Figure S2, Additional file 4:
Figure S3). These regions were removed from further calculations in the PAML software.

\section{Tree topologies}

For each group of invertebrate we aimed at testing the evolutionary mechanism underlying the transition from single- to multi-domain proteins. Although phylogenies including the sequences we studied have been published earlier (Barbatia: Suzuki et al. 1996, nematodes: Blaxter et al. 1994), these often included numerous other sequences. Our topologies only included the orthologs of the multi-domain $\mathrm{Hb}$ from each group and, for comparison, the nearest single-domain globin sequence available for one of the genera that had a multi-domain $\mathrm{Hb}$. Working topologies were generated by maximum likelihood (1,000 bootstrap replications), using MEGA 5 (Nei and Kumar 2000; Tamura et al. 2011) for each of the limited datasets. The best substitution model for each dataset was determined beforehand with jModelTest (Posada 2008) and in all cases the GTR model was selected.

\section{Analyses of selection regimes}

The determination of the selection regimes that acted in the evolution of the multi-domain globins was performed by maximum likelihood analyses on the coding sequences, using the software PAML 4.7 (Yang 1997, 2007). These analyses were also performed with the MEME and REL methods (Murrell et al. 2012), implemented on the Datamonkey.org webserver, to confirm the identification of the sites under selection. Only the PAML method is detailed here, but the methods in Datamonkey also use maximum likelihood algorithms.

The PAML approach uses the codon model of substitutions developed by Goldman and Yang (1994) implemented in Codeml (Nielsen and Yang 1998; Yang 1998; Yang and Nielsen 2002). When using codeml, we chose the mode 'clean data $=1$ ' allowing us to remove all the sites with ambiguous characters and alignment gaps (Yang 1997).

Unrooted tree topologies were used with the condition that single-domain globins or myoglobins form a reciprocal monophyly with respect to the multi-domain globin sequences. At first two branch models, a 'one-ratio' and a 'free-ratio' models, were compared with a likelihood ratio test (LRT), to test whether $\omega$ was homogenous among all lineages $\left(\mathrm{H}_{0}=\right.$ 'one-ratio' model best fits the data, $\mathrm{H}_{1}$ = 'free-ratio' model best fits the data). The LRT is expected to have a $\chi^{2}$ distribution, and the result can then be evaluated in a $\chi^{2}$ table, with the number of degrees of freedom equal to the difference in the number of parameters between the models (Yang 1998). When $\mathrm{H}_{0}$ 
was rejected (i.e. $\omega$ not homogenous amongst lineages), we used a branch-site model (Yang and Nielsen 2002; Zhang et al. 2005), where $\omega$ can vary among codon sites in a foreground lineage when compared to the whole tree (background sites), allowing the identification of amino acid sites under positive selection in that specific branch (Yang and Nielsen 2002). $\mathrm{H}_{0}$ then corresponds to the fitting of the dataset to a nearly-neutral evolution model (M1a in which $\omega$ can be classified into 2 classes: $0<\omega<1$ or $\omega=1$ ), and $\mathrm{H}_{1}$ to the fitting to a model that assumes positive selection among sites of the foreground lineage (Model A (MA) in which $\omega$ can be classified into 3 classes: $0<\omega<1, \omega=1$ and $\omega>1$ ). These two models were compared by LRT and when the MA best fitted the data, we proceeded to one additional comparison; between MA and $\mathrm{MA}_{\omega=1}$ (where $\omega$ is forced to be 1 in the third class) to distinguish relaxed selective constraints from positive selection (Yang and Nielsen 2002; Wong et al. 2004). When this LRT was significant, suggesting the presence of sites under positive selection, the identification of these sites was done by Bayesian analysis, and only sites with a posterior probability greater than $95 \%$ were conserved (Yang 2008). We used the Bayes Empirical Bayes (BEB) analysis preformed by the Codeml package. That method accounts for the sampling errors in maximum likelihood estimates of model parameters (compared to the Naive Empirical Bayes analysis), which could be important in small datasets like ours (Yang et al. 2005).

\section{Sequence-based protein 3D modeling}

The 3D protein models were obtained through the automated protein structure homology-modeling server SWISS-MODEL (Arnold et al. 2006; Kiefer et al. 2009; Peitsch 1995). The quality of the models was assessed through the values of the atomic empirical mean force potential or packing quality of the model (ANOLEA, Melo and Feytmans 1998) and the empirical force field (GROMOS, Eisenberg et al. 1997). These tools measure the goodness of fit between the quaternary structure of the amino acid sequence of interest and the crystal reference used as template. In both cases the more negative the energy values, the more favorable the energy environment. A QMEAN (Benkert et al. 2008) was also used to judge the fitness of the model. This mean is a composite scoring function that accounts at least for the error of residue allocation, comparison with other known 3D models and hypothetical performance of the model through X-ray analyses (cf. SWISS-PROT website). MacPyMOL (PyMol Version 1.3 2010) was used to visualize and edit the produced model, in particular to highlight amino acid residues of interest.

\section{Additional files}

Additional file 1: Figure S1. Alignment of the single-domain and the di-domain globin sequences from the two nematode species. Overall sequence identity (red = low identity, green $=$ high identity, the height of blocks is proportional to the percentage of identity) is represented above the sequences. Please refer to the legend in Figure S2 for the color codes in nucleotide identity and residue hydrophobicity. These sequences were generated from mRNA and for this reason exon limits are not depicted. Alignments were obtained by MUSCLE and submitted to the GUIDANCE filter (more details in the "Methods" section). The regions of the alignments that were not well supported by the filter were removed (shaded areas) from further phylogenetic analyses. Asuum: Ascaris suum, Pdec: Pseudoterranova decipiens, Mg: myoglobin, D: domain.

Additional file 2: Table S1. Identity between orthologs domains (amino acid sequences) from the three analyzed groups of multidomain invertebrate $\mathrm{Hbs}$

Additional file 3: Figure S2. Alignment of the single and tetra-domain globin sequences from the two Polynoidae species. The initial methionine was removed. Overall sequence identity (red = low identity, green $=$ high identity, the height of blocks is proportional to the percentage of identity) is represented above the sequences. The limits of the exons are indicated by the grey bars. Alignments were obtained by MUSCLE and submitted to the GUIDANCE filter (more details in the "Methods" section). The regions of the alignments that were not well supported by the filter were removed (shaded areas) from further phylogenetic analyses. Bsy: Branchipolynoe symmytilida, Bse: B. seepensis, D: domain, SD: single-domain.

Additional file 4: Figure S3. Alignment of the single-domain and the di-domain globin sequences from the mollusk species. Overall sequence identity (red = low identity, green = high identity, the height of blocks is proportional to the percentage of identity) is represented above the sequences. Please refer to the legend in Figure S2 for the color codes in nucleotide identity and residue hydrophobicity. These sequences were generated from mRNA and for this reason exon limits are not depicted. Alignments were obtained by MUSCLE and submitted to the GUIDANCE filter (more details in the "Methods" section). The regions of the alignments that were not well supported by the filter were removed (shaded areas) from further phylogenetic analyses. Bvire: Barbatia virecens, Blima: Barbatia lima, Brev: B. reveeana, Hb: hemoglobin, D: domain.

\section{Abbreviations}

Ma: million years; $\mathrm{Hb}$ : hemoglobin; Mg: myoglobin; SD: single-domain; D: domain; MDa: mega daltons; HBL: exagonal bilayer; LRT: likelihood ratio test; BEB: Bayes Empirical Bayes; REL: random effects likelihood; MEME: mixed effects model of evolution; PAML: phylogenetic analysis by maximum likelihood; PDB: protein data bank; $\omega$ : ratio of nonsynonymous to synonymous mutations $\left(d_{N} / d_{s}\right) ; d_{N}$ : rate of nonsynonymous substitutions; $d_{s}$ : rate of synonymous substitutions.

\section{Authors' contributions}

JPG and SH were responsible for the conception of this study's goal and both drafted this paper. JPG was responsible for data retrieval and all in silico analyses. SH, DJ and SWS participated in the positive selection analyses and $J \mathrm{M}$ in the conception of $3 \mathrm{D}$ models. FHL participated in the draft of this paper and contributed with major revisions. All authors read and approved the final manuscript.

\section{Author details}

${ }^{1}$ CNRS UMR 7144, Station Biologique de Roscoff, Places Georges Teissier, 29680 Roscoff, France. ${ }^{2}$ Laboratoire Adaptation et Diversité en Milieu Marin, UPMC Université Paris 06, Place Georges Teissier, 29680 Roscoff Cedex, France. ${ }^{3}$ Department of Biology and Institute of Molecular Evolutionary Genetics, Pennsylvania State University, University Park, PA 16802, USA. 


\section{Acknowledgements}

This work is part of the project HYPOXEVO (Région Bretagne, France) and was supported by the ESTeam research Marie Curie Grant under the sixth framework program from the European Commission. The authors would also like to acknowledge the anonymous reviewers for their valuable contributions.

\section{Compliance with ethical guidelines}

\section{Competing interests}

The authors declare that there are no competing interests.

\section{Ethical approval}

All reasearch done in this study was based only in silico analyses with data retrieved from genetic databases.

Received: 15 January 2015 Accepted: 29 June 2015

Published online: 16 July 2015

\section{References}

Apic G, Gough J, Teichmann SA (2001) Domain combinations in Archaeal, Eubacterial and Eukariot proteomes. J Mol Biol 310:311-325

Arnold K, Bordoli L, Kopp J, Schwede T (2006) The SWISS-MODEL Workspace: a web-based environment for protein structure homology modelling. Bioinformatics 22:195-201

Bailly X, Leroy R, Carney S, Collin O, Zal F, Toulmond A et al (2003) The loss of the hemoglobin $\mathrm{H} 2 \mathrm{~S}$-binding function in annelids from sulfide-free habitats reveals molecular adaptation driven by Darwinian positive selection. Proc Natl Acad Sci USA 100:5885-5890

Benkert P, Tosatto SCE, Schomburg D (2008) QMEAN: a comprehensive scoring function for model quality assessment. Proteins 71:261-277

Bhaskara RM, Srinivasan N (2011) Stability of domain structures in multidomain proteins. Sci Rep 1:40

Björklund AK, Ekman D, Light S, Frey-Skött J, Elofsson A (2005) Domain rearrangements in protein evolution. J Mol Biol 353:911-923

Blaxter ML (1993) Nemoglobins: divergent nematode globins. Parasitol Today (Personal ed.) 9:353-360

Blaxter ML, Vanfleteren JR, Xia J, Moens L (1994) Structural characterization of an Ascaris myoglobin. J Biol Chem 269:30181-30186

Britten RJ, Kohne DE (1968) Repeated sequences in DNA. Science 161:529-540

Darawshe S, Tsafadyah Y, Daniel E (1987) Quaternary structure of erythrocruorin from the nematode Ascaris suum. Biochem J 242:689-694

De Baere I, Liu L, Moens L, Van Beeumen J, Gielens C, Richelle J et al (1992) Polar zipper sequence in the high-affinity hemoglobin of Ascaris suum: amino acid sequence and structural interpretation. Proc Natl Acad Sci USA 89:4638-4642

De Baere I, Perutz MF, Kiger L, Marden MC, Poyart C (1994) Formation of two hydrogen bonds from the globin to the heme-linked oxygen molecule in Ascaris hemoglobin. Proc Natl Acad Sci USA 91:1594-1597

de Zwaan A, Isani G, Cattani O, Cortesi P (1995) Long-term anaerobic metabolism of erythrocytes arcid clam Scapharca inaequivalvis. J Exp Mar Biol Ecol 187:27-37

Dewilde S, Van Hauwaert M-L, Peeters K, Vanfleteren J, Moens L (1999) Daphnia pulex didomain hemoglobin: structure and evolution of polymeric hemoglobins and their coding genes. Mol Biol Evol 16:1208-1218

Dixon B, Walker B, Kimmins W, Pohajdak B (1991) Isolation and sequencing of a CDNA for an unusual hemoglobin from the parasitic nematode Pseudoterranova decipiens. Proc Natl Acad Sci USA 88:5655-5659

Dixon B, Walker B, Kimmins W, Pohajdak B (1992) A nematode gene contains an intron previously thought to be unique to plants. J Mol Evol 35:131-136

Duboule D, Wilkins AS (1998) The evolution of 'bricolage'. TIG 14:54-59

Edgar RC (2004) MUSCLE: multiple sequence alignment with high accuracy and high throughput. Nucleic Acids Res 32:1792-1797

Eisenberg D, Lüthy R, Bowie JU (1997) VERIFY3D: assessment of protein models with three-dimensional profiles. Methods Enzymol 277:396-404

Eswar N, Marti-Renom MA, Webb B, Madhusudhan MS, Eramian D, Shen M et al (2006) Comparative protein structure modeling with MODELLER. Current protocols in bioinformatics, Suppl 15. Wiley, New Jersey, pp 5.6.1-5.6.30
Flores JF, Hourdez S (2006) The zinc-mediated sulfide-binding mechanism of hydrothermal vent tubeworm 400-kDa hemoglobin. Cah Biol Mar 47:371-377

Flores JF, Fisher CR, Carney SL, Green BN, Freytag JK, Schaeffer SW et al (2005) Sulfide binding is mediated by zinc ions discovered in the crystal structure of a hydrothermal vent tubeworm hemoglobin. Proc Natl Acad Sci USA 102:2713-2718

Gibson QH, Regan R, Olson JS, Carver TE, Dixon B, Pohajdak B et al (1993) Kinetics of ligand to Pseudoterranova decipiens and Ascaris suum hemoglobins and to Leu-29-->; Tyr sperm-whale myoglobin mutant. J Biol Chem 268:16993-16998

Goldman N, Yang Z (1994) A codon-based model of nucleotide substitution for protein-coding DNA sequences. Mol Biol Evol 11:725-736

Grinich NP, Terwilliger RC (1980) The quaternary structure of an unusual highmolecular-weight intracellular haemoglobin from the bivalve mollusc Barbatia reeveana. Biochem J 189:1-8

Grinich NP, Terwilliger RC, Terwilliger NB (1986) Oxygen equilibria and structural characteristics of the tetrameric and polymeric intracellular hemoglobins from the bivalve mollusk Barbatia reeveana. J Comp Physiol B 156:675-682

Han J-H, Batey S, Nickson AA, Teichmann SA, Clarke J (2007) The folding and evolution of multi-domain proteins. Nat Rev Mol Cell Bio 8:319-330

Hoogewiij D, De Henau S, Dewilde S, Moens L, Couvreur M, Borgonie G et al (2008) The Caenorhabditis globin gene family reveals extensive nematode-specific radiation and diversification. BMC Evol Biol 8:279

Hourdez S, Lallier FH, Green BN, Toulmond A (1999a) Hemoglobins from deepsea hydrothermal vent scaleworms of the genus Branchipolynoe: a new type of quaternary structure. Proteins 34:427-434

Hourdez S, Lallier FH, Martin-Jézéquel V, Weber RE, Toulmond A (1999b) Characterization and functional properties of the extracellular coelomic hemoglobins from the deep-sea, hydrothermal vent scaleworm Branchipolynoe symmytilida. Proteins 34:435-442

Hunt PW, McNally J, Barris W, Blaxter ML (2009) Duplication and divergence: the evolution of nematode globins. J Nematol 41:35-51

Jellie AM, Tate WP, Trotman CNA (1996) Evolutionary history of introns in a multi-domain globin gene. J Mol Evol 42:641-647

Kato K, Tokishita S, Mandokoro Y, Kimura S, Ohta T, Kobayahi M et al (2001) Two-domain hemoglobin gene of the water flea Moina macrocopa: duplication in the ancestral Cladocera, diversification, and loss of a bridge intron. Gene 273:41-50

Katoh K, Kuma K-I, Toh H, Miyata T (2005) MAFFT version 5: improvement in accuracy of multiple sequence alignment. Nucleic Acids Res 33:511-518

Kiefer F, Arnold K, Künzli M, Bordoli L, Schwede L (2009) The SWISS-MODEL repository and associated resources. Nucleic Acids Res 37:D387-D392

Kloek AP (1993) Novel gene structure and evolutionary context of Caenorhabditis elegans globin. Gene 129:215-221

Manning AM, Trotman CNA, Tate WP (1990) Evolution of a polymeric globin in the brine shrimp Artemia. Nature 348:653-656

Matthews CM, Vandenberg CJ, Trotman CN (1998) Variable substitution rates of the 18 domain sequences in Artemia hemoglobin. J Mol Evol 1998(46):729-733

Melo F, Feytmans E (1998) Assessing protein structures with a non-local atomic interaction energy. J Mol Biol 277:1141-1152

Minning DM, Goldberg DE (1998) Determinants of Ascaris hemoglobin octamer formation. J Biol Chem 273:32644-32649

Murrell B, Wertheim JO, Moola S, Weighill T, Scheffler K, Kosakovsky Pond SH (2012) Detecting individual sites subject to episodic diversifying selection. PLoS Genet 8(7):e1002764

Naito Y, Riggs CK, Vandergon TL, Riggs AF (1991) Origin of a "bridge" intron in the gene for two domain globin. Proc Natl Acad Sci USA 88:6672-6676

Nei M, Kumar S (2000) Molecular evolution and phylogenetics. Oxford Univ. Press, New York

Nielsen R, Yang Z (1998) Likelihood models for detecting positively selected amino acid sites and applications to the HIV-1 envelope gene. Genetics 148:929-936

Nozawa M, Suzuki Y, Nei M (2009) Reliabilities of identifying positive selection by the branch-site and the site-prediction methods. Proc Natl Acad Sci USA 106:6700-6705

Peitsch MC (1995) Protein modeling by E-mail. Bio/Technology 13:658-660 
Penn O, Privman E, Ashkenazy H, Landan G, Graur D, Pupko T (2010) GUIDANCE; a web server for assessing alignment confidence scores. Nucleic Acids Res 38:W23-W28

Plazzi F, Passamonti M (2010) Towards a molecular phylogeny of Mollusks: Bivalves' early evolution as revealed by mitochondrial genes. Mol Phylogenet Evol 57:641-657

Posada D (2008) jModelTest: phylogenetic model averaging. Mol Biol Evol 25:1253-1256

Projecto-Garcia J, Zorn N, Jollivet D, Schaeffer SW, Lallier FH, Hourdez S (2010) Origin and evolution of the unique tetra-domain hemoglobin from the hydrothermal vent scale worm Branchipolynoe. Mol Biol Evol 27:143-152

PyMol Version 1.3 (2010) PyMOL Molecular Graphics System, Version 1.3.0, Schrödinger, LLC. http://www.pymol.org. Accessed 31 Dec 2010

Riggs AF (1998) Self-association, cooperativity and supercooperativity of oxygen binding by hemoglobins. J Exp Biol 201:1073-1084

Royer WE Jr, Karen SH, Harrington DJ, Chiancone E (1995) The 2.0 A crystal structure of Scapharca tetrameric hemoglobin: cooperative dimers within an allosteric tetramer. J Mol Biol 253:168-186

Royer WE, Knapp JE Jr, Strand K, Heaslet HA (2001) Cooperative hemoglobins: conserved fold, diverse quaternary assemblies and allosteric mechanisms. Trends Biochem Sci 26:297-304

Royer WE Jr, Zhu H, Gorr TA, Flores JF, Knapp JE (2005) Allosteric hemoglobin assembly: diversity and similarity. J Biol Chem 280:27477-27480

Suzuki T, Arita T (1995) Two-domain hemoglobin from the blood clam, Barbatia lima. The cDNA-derived amino acid sequence. J Protein Chem 14:499-502

Suzuki T, Nakamura A, Satoh Y, Inai C, Furukohri T, Arita T (1992) Primary structure of chain I of the heterodimeric hemoglobin from the blood clam Barbatia virescens. J Protein Chem 1:629-633

Suzuki T, Kawasaki Y, Arita T, Nakamura A (1996) Two-domain haemoglobin of the blood clam Barbatia lima resulted from the recent gene duplication of the single-domain $\delta$ chain. Biochem J 313:561-566

Tamura K, Peterson D, Peterson N, Stecher G, Nei M, Kumar S (2011) MEGA5: molecular evolutionary genetics analysis using maximum likelihood, evolutionary distance, and maximum parsimony methods. Mol Biol Evol 28:2731-2739
Vogel C, Teichmann SA, Pereira-Leal J (2005) The relationship between domain duplication and recombination. J Mol Biol 346:355-365

Von Brand T, Baernstein HD, Mehlman B (1950) Studies on the anaerobic metabolism and the aerobic carbohydrate consumption of some fresh water snails. Biol Bull 98:266-276

Voordeckers K, Brown CA, Vanneste K, van der Zande E, Voet A, Maere S et al (2012) reconstruction of ancestral metabolic enzymes reveals molecular mechanisms underlying evolutionary innovation through gene duplication. PLoS Biol 10:e1001446

Weber RE, Vinogradov SN (2001) Nonvertebrate hemoglobins: functions and molecular adaptations. Physiol Rev 81:569-628

Wong WSW, Yang Z, Goldman N, Nielsen R (2004) Accuracy and power of statistical methods for detecting adaptive evolution in protein coding sequences and for identifying positively selected sites. Genetics 168:1041-1051

Yang Z (1997) PAML: a program package for phylogentic analysis by maximum likelihood. Cabios Appl Note Bioinform 13:555-556

Yang Z (1998) Likelihood ratio tests for detecting positive selection and application to primate lysozyme evolution. Mol Biol Evol 15:568-573

Yang Z (2007) PAML 4: phylogenetic analysis by maximum likelihood. Mol Biol Evol 24:1585-1591

Yang Z (2008) Computational molecular evolution. Oxford Univ. Press, New York

Yang Z, Nielsen R (2002) Codon-substitution models for detecting molecular adaptations at individual sites along specific lineages. Mol Biol Evol 19:908-917

Yang Z, Wong WSW, Nielsen R (2005) Bayes empirical Bayes inference of amino acid sites under positive selection. Mol Biol Evol 22:1107-1118

Zal F, Leize E, Lallier FH, Toulmond A, Van Dorsselaer A, Childress JJ (1998) S-Sulfohemoglobin and disulfide exchange: The mechanisms of sulfide binding by Riftia pachyptila hemoglobins. Proc Natl Acad Sci USA 95:8997-9002

Zhang J, Nielsen R, Yang Z (2005) Evaluation of an improved branch-site likelihood method for detecting positive selection at the molecular level. Mol Biol Evol 22:2472-2479

\section{Submit your manuscript to a SpringerOpen ${ }^{\circ}$ journal and benefit from:}

- Convenient online submission

- Rigorous peer review

- Immediate publication on acceptance

Open access: articles freely available online

- High visibility within the field

- Retaining the copyright to your article

Submit your next manuscript at springeropen.com 